\title{
Oxyresveratrol inhibits the growth of human lung squamous cell carcinoma cells by triggering S-phase arrest and apoptosis
}

\author{
Cheng-Hsuan Chuang ${ }^{\mathrm{a}}$, Kok-Tong Tan ${ }^{\mathrm{a}, \mathrm{b}}$, Yu-Tang Tung ${ }^{\mathrm{c}}$ and Chi-Chien Lin ${ }^{\mathrm{a}, \mathrm{d}}$
}

\begin{abstract}
anstitute of Biomedical Science, National Chung-Hsing University, Taichung 402, Taiwan
'Tungs' Taichung MetroHarbor Hospital, Taichung 404, Taiwan

${ }^{\mathrm{c}}$ Graduate Institute of Metabolism and Obesity Sciences, Taipei Medical University, Taipei 11031, Taiwan

${ }^{\mathrm{d} D e p a r t m e n t}$ of Medical Research, China Medical University Hospital, Taichung 404, Taiwan

${ }^{*}$ Corresponding author: Chi-Chien Lin, Institute of Biomedical Science, National Chung-Hsing University, 50 Kuo-Kuang Road, Taichung 40227, Taiwan, R.O.C. Tel: +886-4-2284089; E-mail: lincc@dragon.nchu.edu.tw; Yu-Tang Tung, Graduate Institute of Metabolism and Obesity Sciences, Taipei Medical University, Taiwan, 250 Wu-Hsing Road, Taipei 11031, Taiwan, R.O.C. Tel: +886-2-27361661 ext. 7529; E-mail: f91625059@ntu.edu.tw
\end{abstract}

DOI: $10.31665 /$ JFB.2019.6190

Received: June 19, 2019; Revised received \& accepted: June 30, 2019

Citation: Chuang, C.-H., Tan, K.-T., Tung, Y.-T., and Lin, C.-C. (2019). Oxyresveratrol inhibits the growth of human lung squamous cell carcinoma cells by triggering S-phase arrest and apoptosis. J. Food Bioact. 6: 131-139.

\begin{abstract}
Oxyresveratrol is found in mulberry (Morus alba L.) and possesses antioxidant, anti-inflammatory, and antitumor activities. However, to the best of our knowledge, relevant studies on the effects of oxyresveratrol on human lung squamous cell carcinoma cells are lacking. The aim of this study was to evaluate the antitumor activity of oxyresveratrol in human $\mathrm{NCl}-\mathrm{H} 520$ lung squamous cell carcinoma cells using in vitro and in vivo approaches. Oxyresveratrol induced $\mathrm{NCl}-\mathrm{H} 520$ cell apoptosis in an intrinsic signaling pathway. Therefore, oxyresveratrol resulted in enhanced cell numbers at the sub- $G_{1}$ phase, increased caspase- 3 and -9 activities, caused the MMP (mitochondrial membrane potential) to collapse, and decreased Bcl-2 and cyclin D protein expressions. In addition, oxyresveratrol induced arrest at the S-phase cell cycle in $\mathrm{NCl}-\mathrm{H} 520$ cells through downregulating the cell-cycle-related protein expressions of cyclin A, cyclin B, and cdk2. These results indicated that oxyresveratrol inhibited the proliferation and induced apoptosis by activating an intrinsic pathway in $\mathrm{NCl}-\mathrm{H} 520$ non-small cell lung cancer cells. Our results suggest that oxyresveratrol can be used as an alternative remedy for human non-small-cell lung cancer.
\end{abstract}

Keywords: Oxyresveratrol; Non-small cell lung cancer; Cell cycle arrest; Apoptosis; Cell cycle-related proteins.

\section{Introduction}

Lung cancer (both small cell and non-small cell) is the second most common cancer in both men and women. In 2017 in the United States, lung cancer was the most common cause of cancer deaths among both men and women; about one of four cancer deaths (approximately 155,870 deaths including 84,590 in men and 71,280 in women) were due to lung cancer according to the American Cancer Society's estimates. Among them, non-small cell lung cancers (NSCLCs) account for over $85 \%$ of lung cancers, and of these, approximately $30 \%$ are squamous cell carcinomas (SCCs) (Heist et al., 2012). In addition, SCC comprises $91 \%$ of cases of lung cancer due to cigarette smoking (Molina et al., 2012; Alberg et al., 2005; Ettinger a et al., 2013). Despite major advances in cancer therapeutics, survival rates for lung cancer patients have not yet improved significantly, even in the targeted therapy era (Sant et al., 2003). It is clear that traditional chemotherapeutic agents have reached a therapeutic plateau (Carney, 2003), and there is a need for tumor prevention in addition to more-effective treatments (Toh et al., 2006).

Oxyresveratrol (trans-2,30,4,50-tetrahydroxystilbene), which has one more hydroxyl group than resveratrol, is a natural hydroxystilbene found in the mulberry (Morus alba L.). Morus plants, a common food and herbal medicine in China, are used as a tradi- 
tional remedy to cure inflammatory diseases (Chen et al., 2013). Research has shown that oxyresveratrol has numerous biological activities that include anti-cyclooxygenase (Shin et al., 1998), anti-rat liver mitochondrial ATPase (Nimmanpisut et al., 1976), anti-DOPA oxidase (Song et al., 2007), antitumor (Li et al., 2010; Mouihate et al., 2006), antiviral (Galindo et al., 2011; Jagtap et al., 2010), and neuroprotective activities (Breuer et al., 2006). Previous studies have shown that oxyresveratrol inhibits protein kinase $\mathrm{C}$ (PKC), which likely serves as a receptor for tumor promoters (Hu et al., 1996). Li et al. (2010) pointed out that oxyresveratrol together with resveratrol enhanced the cytotoxicity of HT-29 human colon cancer cells and also found that oxyresveratrol had about 2-fold more-potent cytotoxic activity than resveratrol. In addition, $\mathrm{Wu}$ et al. (2010) indicated that oxyresveratrol exhibited in vitro cytotoxicity against a human breast cancer cell line.

However, to the best of our knowledge, the effects of oxyresveratrol on human lung SCC cells still lack support from the relevant scientific literature. Hence, the aims of this study were to evaluate the antitumor activity of oxyresveratrol in human lung SCC NCIH520 cells using in vitro and in vivo approaches, and determine its mechanisms of action. To obtain insights into its mechanism of action, the effects of oxyresveratrol on cell proliferation, the cell-cycle distribution, apoptosis, and expression levels of several cell-cycle- and apoptosis-related proteins were examined.

\section{Material and methods}

\subsection{Cell culture}

Human lung SCC NCI-H520 cells were obtained from the Bioresource Collection and Research Center (BCRC 60124) and cultured in RPMI 1640 medium supplemented with $10 \%(\mathrm{v} / \mathrm{v})$ fetal bovine serum (FBS; Gibco, Grand Island, NY) and 1\% (v/v) antibiotic-antimycotic (Gibco). Cells were incubated in a humidified incubator (with $5 \% \mathrm{CO}_{2}$ and $95 \%$ air) at $37{ }^{\circ} \mathrm{C}$.

\subsection{Cell viability assay}

The 3-(4,5-dimethylthiazol-2-yl)-2,5-diphenyltetrazolium (MTT, Sigma-Aldrich, St. Louis, MO, USA) test is a method for detecting cell viability using a colorimetric assay. To measure the cytotoxicity of oxyresveratrol (Sigma-Aldrich) against cell proliferation, NCI-H520 cells were seeded overnight into a 24-well plate in triplicate to allow cell adherence. First, $200 \mu \mathrm{L}$ of fresh medium containing various concentrations of $0.1 \%$ DMSO (Control) or oxyresveratrol $(6.25,12.5,25,50$, and $100 \mu \mathrm{M})$ was added to the culture plate and incubated at $37^{\circ} \mathrm{C}$ for $24 \mathrm{~h}$. Following removal of the medium from the wells, $200 \mu \mathrm{L}$ of the MTT reagent $(5 \mathrm{mg} / \mathrm{mL})$ was added. After $4 \mathrm{~h}$ of incubation at $37{ }^{\circ} \mathrm{C}$, the medium was removed, and $600 \mu \mathrm{L}$ of DMSO was added to dissolve the formazan crystals. The absorbance was measured at $540 \mathrm{~nm}$ using a microplate reader (Tecan Sunrise). The cell viability ratio (\%) was calculated from the following equation: $\%$ viability $=($ absorbance of test sample / absorbance of control) x 100\%.

\subsection{Cell-cycle distribution analysis}

Cells were incubated with $0.1 \%$ DMSO or various concentrations $(25,50$, or $100 \mu \mathrm{M})$ of oxyresveratrol for $24 \mathrm{~h}$. After treatment, cells were trypsinized and fixed in ice-cold $80 \%$ ethanol, and then stored overnight at $-20{ }^{\circ} \mathrm{C}$. Next, cells were washed three times in phosphate-buffered saline (PBS) and incubated with $50 \mu \mathrm{g} / \mathrm{mL}$ PtdIns and $25 \mu \mathrm{g} / \mathrm{mL}$ RNase A (BD Bioscience, San Jose, CA) for $30 \mathrm{~min}$ at room temperature in the dark. These stained cells were immediately analyzed using a Accuri 5 flow cytometer (Accuri Cytometers) equipped with C6 Accuri system software (Accuri Cytometers). All experiments were performed in triplicate and yielded similar results.

\subsection{Annexin V-FITC binding assay}

An apoptosis assay was performed using the Annexin V-FITC Apoptosis Detection Kit (BioVision), according to the manufacturer's instruction. Cells were treated with $0.1 \% \mathrm{DMSO}$ or various concentrations $(25,50$, or $100 \mu \mathrm{M})$ of oxyresveratrol for $24 \mathrm{~h}$. Cells were trypsinized, washed twice with PBS, and subsequently resuspended in $500 \mu \mathrm{L}$ of $1 \times$ binding buffer. The cell suspension was then incubated with the cultures and stained with $5 \mu \mathrm{L}$ of FITClabeled Annexin $\mathrm{V}$ and $5 \mu \mathrm{L}$ of propidium iodide (PI) for $15 \mathrm{~min}$ at room temperature in the dark. These stained cells were immediately analyzed using a Accuri 5 flow cytometer (Accuri Cytometers) equipped with C6 Accuri system software (Accuri Cytometers). All experiments were performed in triplicate and yielded similar results.

\subsection{Western blot analysis}

Protein expression levels of cell cycle-related proteins (cyclin A, cyclin B, cyclin D, and cdk2) and apoptosis-related proteins (Bcl2 and Bax) in cells were determined using a Western blot analysis according to a method previously described by Li et al. (2016). In this study, the primary antibodies were anti-cyclin A (clone BF683, CAS: 4656, Cell Signaling), anti-cyclin B (clone GNS1, CAS: sc-245, Santa Cruz Biotechnology), anti-cyclin D (clone EPR2241(IHC)-32, CAS: 2261-1, Epitomics), anti-cdk2 (clone E304, CAS: 1134-1, Epitomics), anti-Bcl-2 (clone 50E3, CAS: 2870, Cell Signaling), anti-Bax (clone polyclonal, CAS: 2772, Cell Signaling), and anti- $\beta$-actin (clone C4, CAS: sc47778, Santa Cruz).

\subsection{Measurement of the mitochondrial membrane potential (MMP)}

The MMP was measured using a JC-1 mitochondrial-specific fluorescent probe (Invitrogen, Carlsbad, CA, USA) based on the method of Li et al. (2016). Cells were treated with $0.1 \%$ DMSO or various concentrations $(25,50$, or $100 \mu \mathrm{M})$ of oxyresveratrol for $24 \mathrm{~h}$, and then stained with $10 \mathrm{mg} / \mathrm{mL}$ of JC- 1 at $37{ }^{\circ} \mathrm{C}$ for $10 \mathrm{~min}$. Cells were washed twice with PBS, and cells with an FL-1 (530-nm emission) versus FL-2 (580-nm emission) dot plot were quantified using an Accuri 5 flow cytometer (Accuri Cytometers) equipped with C6 Accuri system software (Accuri Cytometers).

\subsection{Caspase- $-3,-8$, and -9 activity assay}

After treatment with $0.1 \%$ DMSO or various concentrations (25, 50 , or $100 \mu \mathrm{M}$ ) of oxyresveratrol for $24 \mathrm{~h}$, cells were collected for measurement of caspase-3, -8 , and -9 activities using the appropriate CaspGLOW ${ }^{\mathrm{TM}}$ Fluorescein Active Caspase Staining Kits 
(Biovision, Milpitas, CA, USA), and the protocols were performed according to the manufacturer's instructions.

\subsection{In vivo antitumor activity}

Animal procedures were approved by the Institutional Animal Care and Use Committee (IACUC no. 106-046) of National Chung Hsing University. NCI-H520 cells were mixed with Matrigel (BD Biosciences) at a 1:1 ratio. Cells $\left(10^{7}\right)$ were subcutaneously injected into the back of nude mice, and tumors were allowed to grow for 8 days until they reached $10 \mathrm{~mm}^{3}$, and then they were treated with either the vehicle $(10 \% \mathrm{DMSO}+90 \%$ glyceryl trioctanoate) or $250 \mathrm{mg} / \mathrm{kg}$ of oxyresveratrol (dissolved in DMSO and glyceryl trioctanoate, 10:90 v/v) in an intraperitoneal (i.p.) injection using $100 \mathrm{~mL}$ total volume for 3 weeks. Tumor-bearing mice were sacrificed after 30 days. The tumor volume was measured daily, and it was estimated using the formula: length $\mathrm{x}$ width $\mathrm{x}$ thickness $\mathrm{x} 0.5$ and is expressed in $\mathrm{mm}^{3}$. Tumors were fixed in $4 \%$ formaldehyde for $24 \mathrm{~h}$, subsequently paraffin-embedded, sectioned into $6-\mu \mathrm{m}$-thick sections, and examined using hematoxylin and eosin (H\&E) staining.

\subsection{Statistical analysis}

Data are expressed as the mean \pm standard deviation (SD). An analysis of variance (ANOVA) was employed to calculate differences between multiple groups with Duncan's test. A $p$ value $<$ 0.05 was considered statistically significant.

\section{Results}

\subsection{Oxyresveratrol inhibits the proliferation of $\mathrm{NCl}-\mathrm{H} 520$ cells and induced $S$ cell-cycle arrest and apoptosis in those cells}

To investigate the potential cell proliferation-inhibitory activity of oxyresveratrol in human lung SCC, NCI-H520 cells were incubated in the absence and presence of various concentrations of oxyresveratrol $(6.25 \sim 100 \mu \mathrm{M})$ for $24 \mathrm{~h}$, and cell viability was determined by an MTT assay. As shown in Figure 1a, 25, 50, and $100 \mu \mathrm{M}$ oxyresveratrol significantly inhibited the proliferation of NCI-H520 cells in a dose-dependent manner. To further examine whether oxyresveratrol causes cell death by cell-cycle arrest and/ or apoptosis, NCI-H520 cells were treated with oxyresveratrol at 25,50 , or $100 \mu \mathrm{M}$ for $24 \mathrm{~h}$, and then the DNA contents of cells were quantitated by flow cytometry of cells stained with PI. PI staining was performed to determine whether oxyresveratrolmediated cell apoptosis and/or proliferation inhibition was due to cell-cycle arrest (Figure 1b). PI staining revealed that oxyresveratrol enhanced cell numbers in the sub- $\mathrm{G}_{1}$ phase, where hypodiploid $(\leq 2 \mathrm{~N})$ fragmented DNA is a marker of apoptosis (Karna et al., 2011). Data were confirmed by an Annexin V-based apoptosis assay. As shown in Figure 1c, the percentage of Annexin V-positive staining indicates that 50 and $100 \mu \mathrm{M}$ oxyresveratrol increased early and late apoptotic cells in NCI-H520 cells. Moreover, oxyresveratrol also led to the accumulation of cells in the S-phase and was associated with a concomitant decrease of cells in the $\mathrm{G}_{1}$ phase in a dose-dependent manner (Figure 1b). Thus, results showed that oxyresveratrol-mediated cell apoptosis and proliferation inhibition (S-phase arrest) were due to cell-cycle arrest in NCI-H520 cells.

\subsection{Oxyresveratrol affects cell cycle-associated protein expres- sions in $\mathrm{NCl}-\mathrm{H} 520$ cells}

To further investigate the cell-cycle arrest of NCI-H520 cells mediated by oxyresveratrol, cell cycle-related proteins were examined. CDK and cyclin control the cell cycle (Tan et al., 2011). In order to examine modulators involved in the early cell cycle, such as in the sub- $\mathrm{G}_{1}$ phase, cyclin $\mathrm{D}$ was detected. As shown in Figure 2, oxyresveratrol suppressed cyclin D. This finding suggests that oxyresveratrol inhibited progression of the $G_{0} / G_{1}$ phase and resulted in enhanced cell numbers in the sub- $\mathrm{G}_{1}$ phase (Figure 1b). Because oxyresveratrol induced S-phase cell-cycle arrest in NCI-H520 cells (Figure 1b), this study investigated whether the $\mathrm{G}_{2}$-phase cyclin, cyclin $\mathrm{B} 1$, was affected by oxyresveratrol. Cells were treated with 25,50 , or $100 \mu \mathrm{M}$ of oxyresveratrol, and levels of cyclin B1 expression were analyzed at $24 \mathrm{~h}$ after treatment (Figure 2). Cyclin B1 expression decreased after treatment with oxyresveratrol, which is consistent with S-phase arrest. This fairly uniform decrease in cyclin B1 could explain the ability of oxyresveratrol to inhibit $\mathrm{G}_{2}$-phase entry. In addition, cyclin $\mathrm{A}$ and $\mathrm{CDK} 2$ are associated with the $\mathrm{S}$ phase of cells. CDC25A activates CDK2, which in turn activates the cyclin-CDK complex. The cyclin-CDK complex promotes operation of the cell cycle and therefore causes rapid cell growth (George Rosenker et al., 2015; Tilaoui et al., 2014). Figure 2 shows that oxyresveratrol reduced expressions of cyclin A and CDK2 proteins (Figure 3). Therefore, oxyresveratrol decreased the proliferation by arresting NCI-H520 cells in the $\mathrm{S}$ phase.

\subsection{Oxyresveratrol induces apoptosis primarily via the intrinsic mitochondrial pathway and modulates Bcl-2 family protein expressions in $\mathrm{NCl}-\mathrm{H} 520$ cells}

To investigate whether the mitochondrial pathway is involved in oxyresveratrol-induced apoptosis of NCI-H520 cells, changes in the MMP $\left(\Delta \Psi_{\mathrm{m}}\right)$ and protein expressions of Bcl-2 and Bax were analyzed. Changes in the MMP were detected using JC-1, a mitochondrial-specific fluorescent probe. The red fluorescence of JC-1 indicates intact mitochondria, whereas green fluorescence indicates that the monomeric JC-1 remains unprocessed owing to collapse of the MMP (Kwon et al., 2011).

As shown in Figure 4a, oxyresveratrol caused a dose-dependent increase in green fluorescence and a concomitant attenuation in red fluorescence in NCI-H520 cells, indicating that oxyresveratrol triggered a reduction in the MMP. The Bcl-2 family is made up of outer mitochondrial membrane proteins that can regulate apoptosis by the antiapoptotic protein, Bcl-2, or the proapoptotic protein, Bax, in the cell death process (Levy and Claxton, 2017). Therefore, we analyzed the effects of oxyresveratrol on Bax and Bcl-2 expressions in NCI-H520 cells. As shown in Figure 4b, oxyresveratrol resulted in a significant decrease in the expression of the antiapoptotic protein, Bcl-2, while expression of the proapoptotic Bax protein was not significantly affected. However, the Bcl-2/Bax ratio significantly decreased, which suggests that oxyresveratrol induced apoptosis of NCI-H520 cells via a mitochondrial apoptotic pathway.

\subsection{Oxyresveratrol induces apoptosis through a caspase path- way in NCl-H520 cells}

Caspases involved in apoptosis are generally divided into two 
a
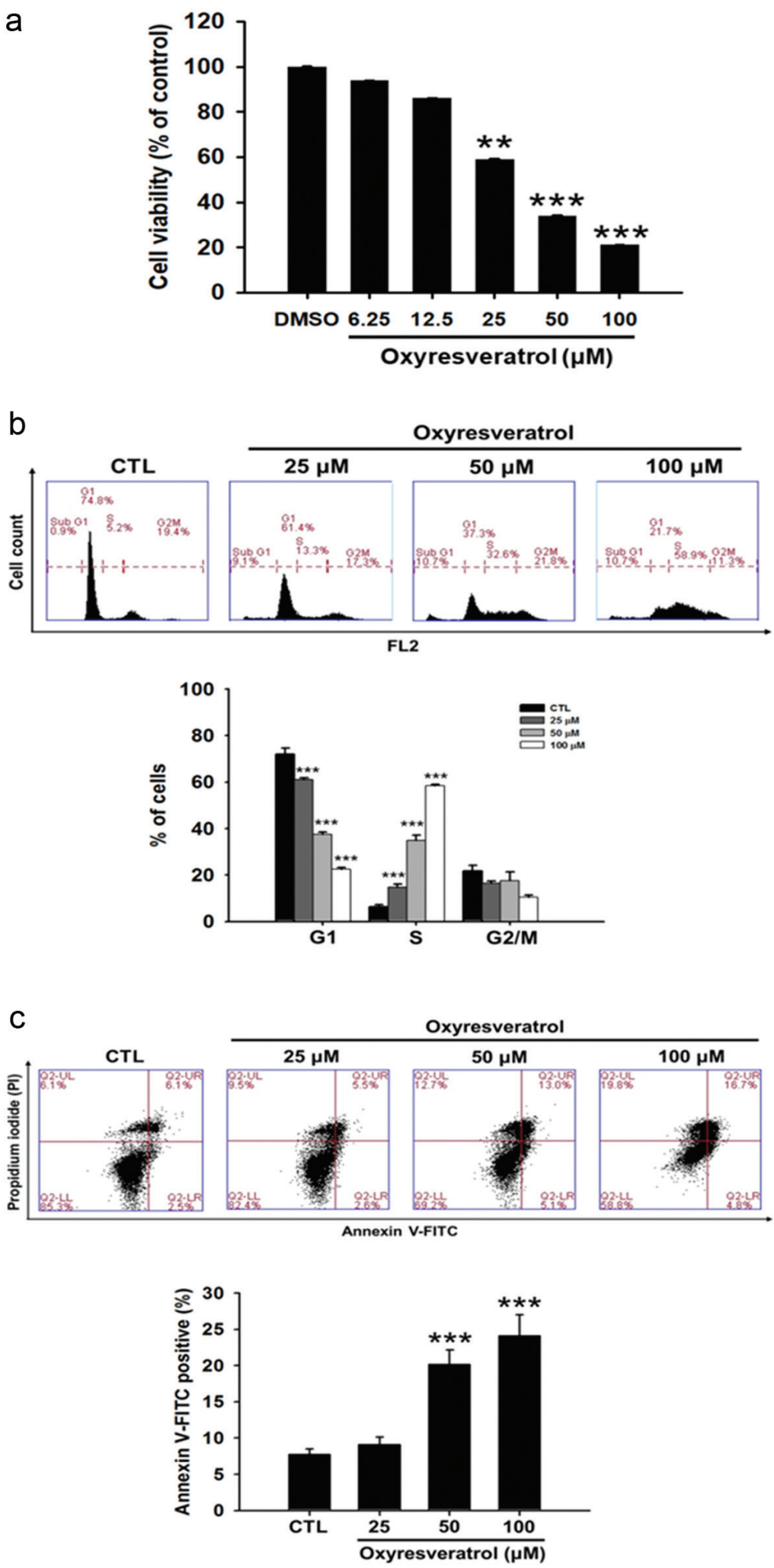

Figure 1. Effects of oxyresveratrol on (a) cytotoxicity, (b) cell cycle progression, and (C) apoptosis of $\mathrm{NCl}-\mathrm{H} 520$ cells. $\mathrm{NCl}-\mathrm{H} 520$ cells were treated with oxyresveratrol for $24 \mathrm{~h}$. The cytotoxicity was analyzed by an MTT assay, and cell cycle distributions were then analyzed by flow cytometry. Apoptosis in NClH520 cells was examined by Annexin V-FITC/propidium iodide (PI) binding and analyzed by flow cytometry. Data are presented as the mean \pm SD of three experiments each conducted in triplicate. ${ }^{* *} p<0.01$ and ${ }^{* * *} p<0.001$ compared to the DMSO-treated group. 


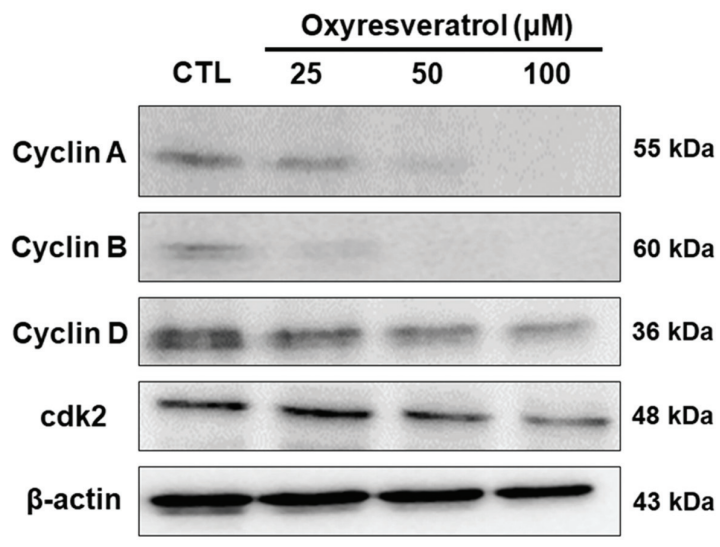

Figure 2. Effects of oxyresveratrol on cell-cycle-related proteins in $\mathrm{NCl}-$ H520 cells. NCl-H520 cells were treated with 25,50 , or $100 \mu \mathrm{M}$ oxyresveratrol for $24 \mathrm{~h}$, and then whole-cell lysates were prepared and subjected to Western blotting using antibodies against the indicated proteins. $\beta$-Actin was used as a loading control. Bands were analyzed by ImageJ software (National Institutes of Health) and normalized to $\beta$-actin

categories: initiator caspases (such as caspases-2, -8, -9, and -10) and effector caspases (such as caspases-3, -6, and -7) (Shi, 2002). Caspases- 8 and -9 can initiate downstream protease activity, while the downstream one in the implementation of apoptosis, caspase-3, can destroy cellular proteins and DNA. NCI-H520 cells were treated with 25,50 , or $100 \mu \mathrm{M}$ oxyresveratrol for $24 \mathrm{~h}$, and then caspase- $3,-8$, and -9 activities were quantitated by flow cytometry. Figure 4 indicates that oxyresveratrol significantly increased caspase- $3,-8$, and -9 activities in dose-dependent manners. Therefore, treatment with oxyresveratrol can activate caspases-3, -8 and -9 , thereby leading to apoptosis.

\subsection{Oxyresveratrol inhibits $\mathrm{NCl}-\mathrm{H} 520$ tumor growth in nude mice}

To validate our in vitro results, the anticancer effect of oxyresveratrol in human lung SCC xenograft mice was evaluated. NCIH520 cells were subcutaneously injected into the back of nude mice. Oxyresveratrol at $250 \mathrm{mg} / \mathrm{kg}$ via an i.p injection significantly inhibited tumor growth from days 17 to 30 compared to vehicle-treated mice (Figure $5 \mathrm{~b}$ ). Notably, treatment of mice with oxyresveratrol may have led to some degree of harm to the experimental mice, but in this study no mice exhibited obvious abnormal behaviors or died for non-tumor factors, suggesting that this dose of oxyresveratrol was not toxic for i.p. injections in mice. Moreover, we measured the wet weights of the tumors, and results showed that oxyresveratrol significantly reduced tumor weights compared to vehicle-treated mice (Figure $5 \mathrm{c}$ ). These results in-
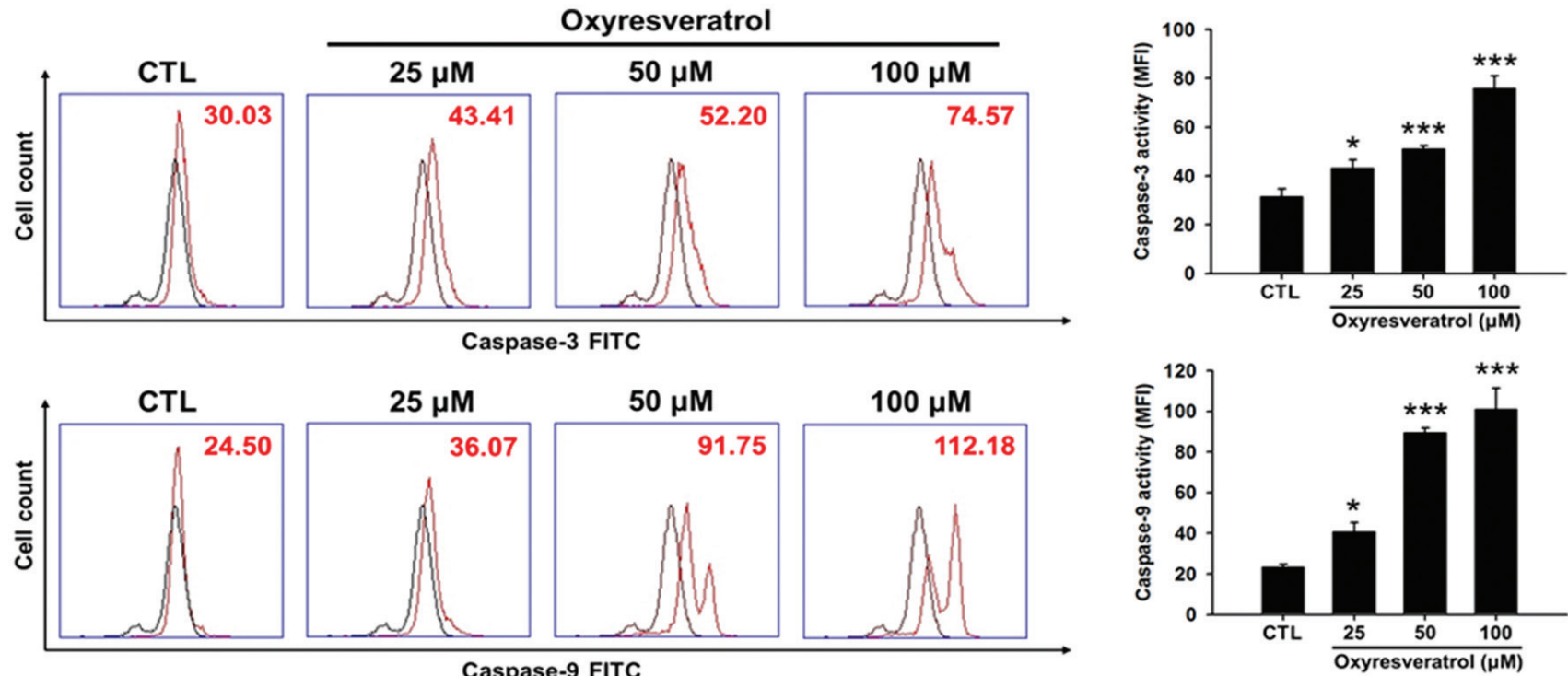

Caspase-9 FITC
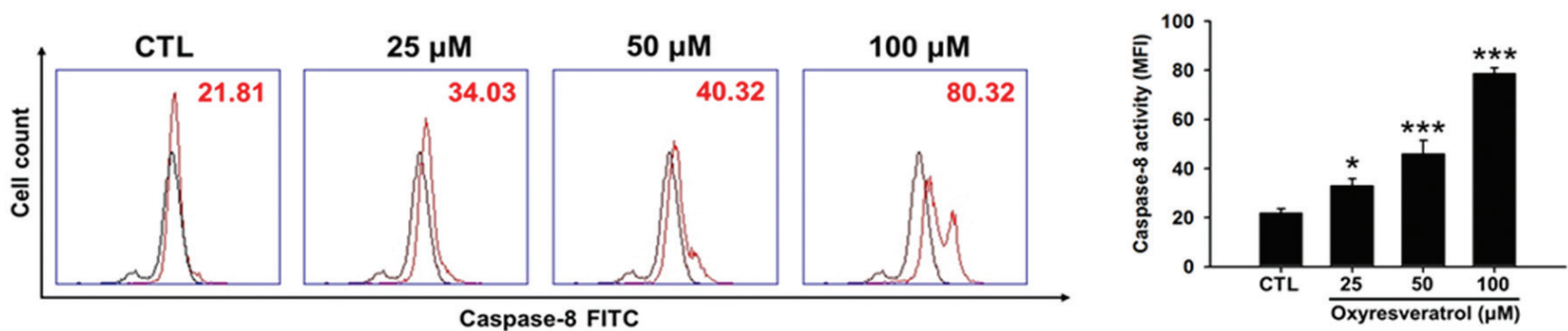

Figure 3. Effects of oxyresveratrol on caspase-3, -8 , and -9 activities in $\mathbf{N C l}-\mathrm{H} 520$ cells. $\mathrm{NCl}-\mathrm{H} 520$ cells were treated with 25,50 , or $100 \mu \mathrm{M}$ oxyresveratrol for $24 \mathrm{~h}$ and then the activities of caspases-3, -8 , and -9 were determined by flow cytometry. Black line: mock control of DMSO-treated cell control; red line: cells treated with oxyresveratrol. Data are presented as the mean \pm SD of three experiments each conducted in triplicate. ${ }^{*} p<0.05,{ }^{* *} p<0.01$, and ${ }^{* * *} p<$ 0.001 compared to the DMSO-treated group. 
a
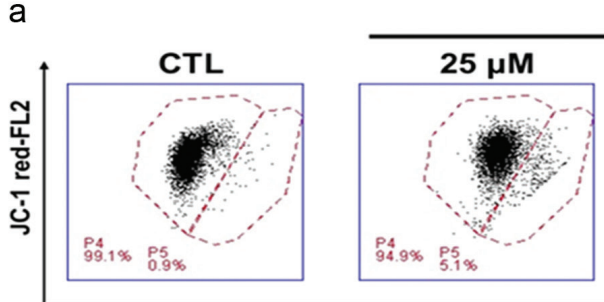

Oxyresveratrol

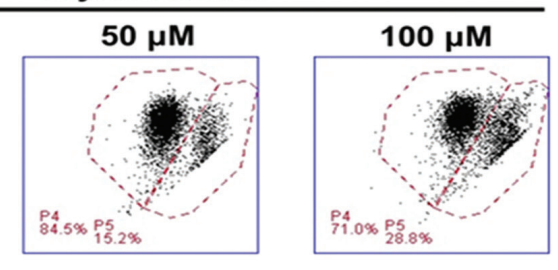

JC-1 green-FL1

b

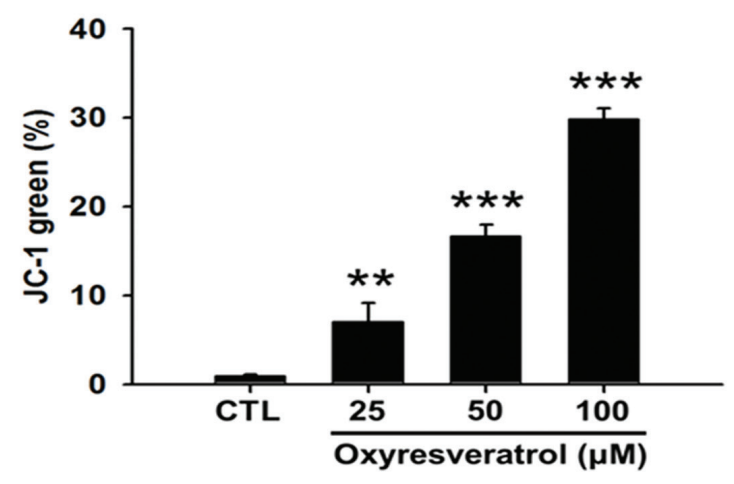

C

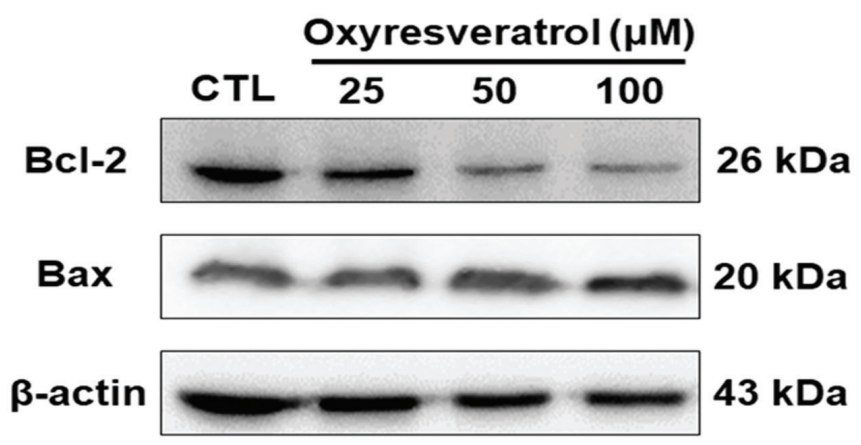

Figure 4. Effect of oxyresveratrol on the mitochondrial pathway of apoptosis in $\mathrm{NCl}-\mathrm{H} 520$ cells. $\mathrm{NCl}-\mathrm{H} 520$ cells were treated with 25,50 , or $100 \mu \mathrm{M}$ oxyresveratrol for $24 \mathrm{~h}$. (a) Disruption of the mitochondrial membrane potential. Data are presented as the mean \pm SD of three experiments each conducted in triplicate. ${ }^{* *} p<0.01$ and ${ }^{* * *} p<0.001$ compared to the DMSO-treated group. (b) Bcl-2 and Bax were examined by a protein gel blot analysis. $\beta$-Actin was used as a loading control. Bands were analyzed by ImageJ software (National Institutes of Health) and normalized to $\beta$-actin.

dicate that oxyresveratrol also inhibited the growth of small lung cancer cells in vivo.

\section{Discussion}

Polyphenols and flavonoids are abundant in plants. Previous studies have demonstrated that they have potential uses in treating or preventing cancer, and therefore they may be chemopreventive and antiproliferative agents(Shukla et al., 2014; Priyadarsini, and Nagini, 2012; Surh, 2003; Yang et al, 2001; Watson et al, 2000; Middleton et al , 2000). Oxyresveratrol (trans-2,30,4,50-tetrahydroxystilbene), a natural hydroxystilbene, is present in Morus alba (mulberry) (Lorenz et al., 2003). Moreover, pharmacological studies showed that oxyresveratrol given orally has a bioavailability of about 50\% (Qiu et al., 1996). Oxyresveratrol is a naturally occurring oxidative analog of resveratrol (Ban et al., 2006). Similar to resveratrol, oxyresveratrol possesses many beneficial pharma- cological activities such as antitumor ( $\mathrm{Li}$ et al., 2010; Mouihate et al., 2006), antiviral (Galindo et al., 2011; Jagtap et al., 2010), antioxidant (Lorenz et al., 2003), hepatoprotective (Ban et al., 2006), COX-inhibitory (Shin et al., 1998; Su et al , 2002), and neuroprotective activities (Breuer et al., 2006). In addition, oxyresveratrol can enhance the immune system (Xu et al., 2014) and can lower blood fat (Xu et al., 2014) and blood sugar (Xu et al., 2014) levels. Hu et al. (Hu et al., 1996) showed that oxyresveratrol inhibits protein kinase $\mathrm{C}$, which probably serves as a receptor for tumor promoters, and also found that oxyresveratrol showed non-competitive inhibition (Nishizuka, 1984). Likewise, Li et al. (2010) pointed out cell proliferation-inhibitory activities of oxyresveratrol and resveratrol in HT-29 human colon cancer cells and found the cytotoxic activity of oxyresveratrol to be about 2-fold greater than that of resveratrol. Furthermore in an in vitro assay, Wu et al. (2010) reported that oxyresveratrol has anti-breast tumor cell activity. However, to the best of our knowledge, there has been no study focusing on the effects of oxyresveratrol on human lung 

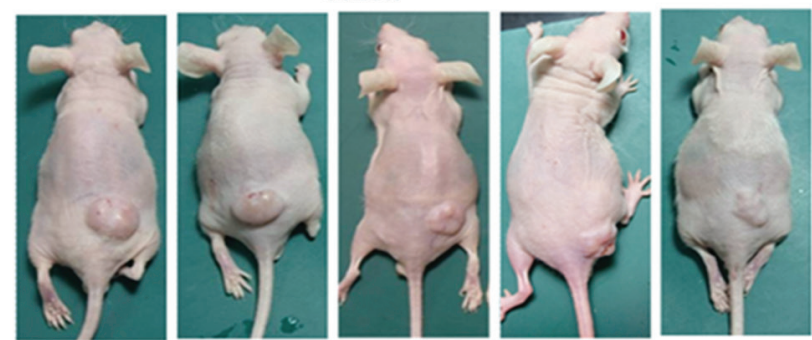

\section{Oxyresveratrol $(250 \mathrm{mg} / \mathrm{kg})$}
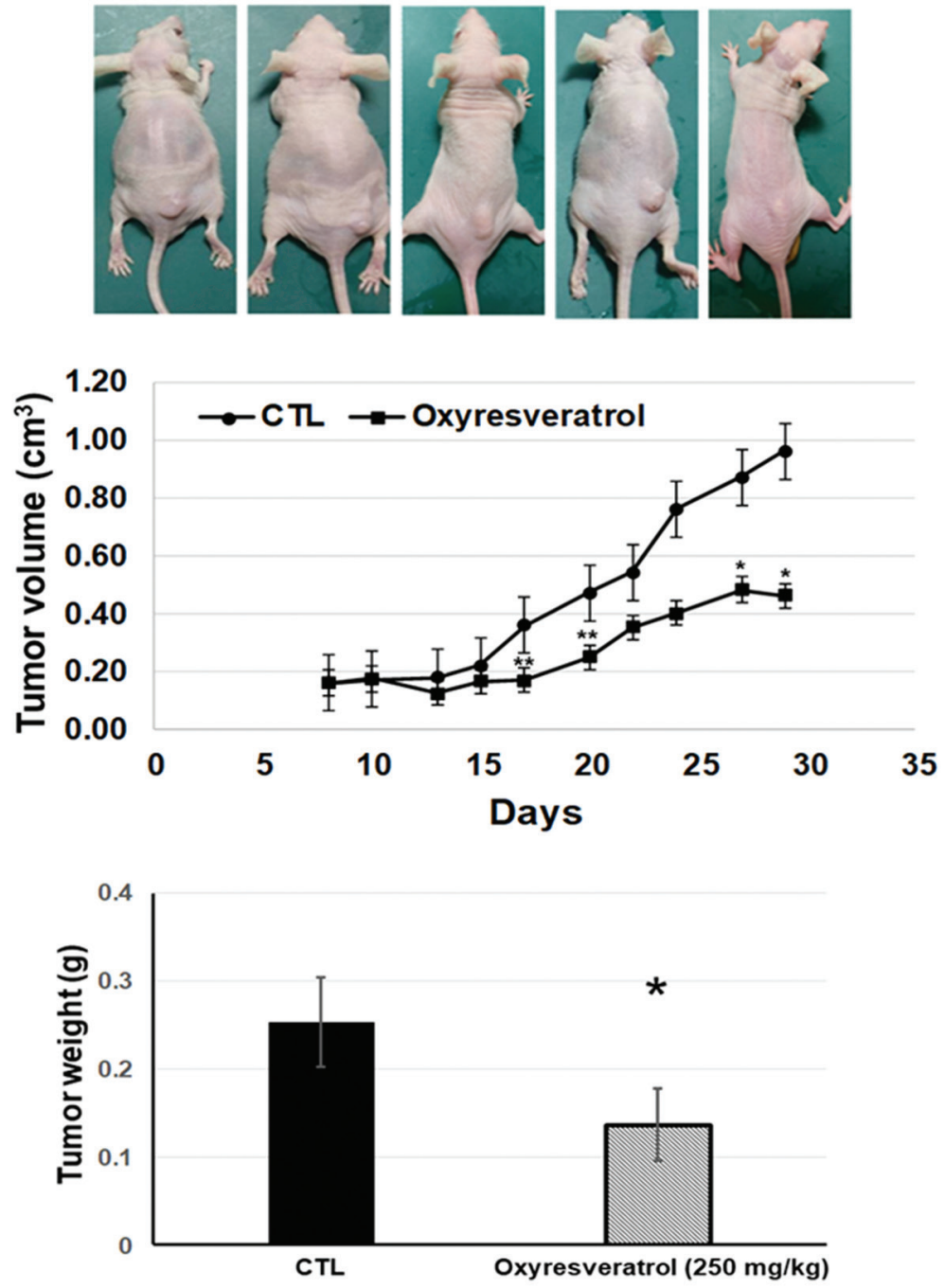

Figure 5. Oxyresveratrol inhibited $\mathrm{NCl}-\mathrm{H} 520$ tumor growth in nude mice. $\mathrm{NCl}-\mathrm{H} 520$ cells $\left(10^{7}\right)$ were subcutaneously injected into mice and allowed to grow up to approximately $10 \mathrm{~mm}^{3}$ in size. Oxyresveratrol $(250 \mathrm{mg} / \mathrm{kg})$ and a vehicle control were administered intraperitoneally daily for three weeks until day 30 $(n=5)$. Photograph of tumor tissues (a), the time course of tumor sizes (b), and the tumor weight on day 30 . Quantified data are presented as the mean \pm $\mathrm{SD}(n=5) .{ }^{*} p<0.05$ and ${ }^{* *} p<0.01$ of comparisons between the vehicle and oxyresveratrol groups.

\section{SCC cells.}

In this study, we first found that oxyresveratrol suppressed the growth of NCI-H520 cells in a dose-dependent manner $(p<0.05)$ (Figure 1a). Furthermore, we also demonstrated that the ability of oxyresveratrol to suppress cancer cell growth was mainly achieved through the mechanisms of apoptosis and suppressing cell prolif- eration. The effect of oxyresveratrol on the cell cycle was studied, and it was found that oxyresveratrol arrested NCI-H520 cells at the sub- $\mathrm{G}_{1}$ and $\mathrm{S}$ phases (Figure $1 \mathrm{~b}$ ). Furthermore, oxyresveratrol caused an increase in cells in the $\mathrm{S}$ phase and a corresponding decrease of cells in the $\mathrm{G}_{1}$ phase. To further confirm the S-cell cycle arrest mediated by oxyresveratrol, S-cell cycle-related proteins 
(cyclin A and cdk2) were examined (Figure 2). We also found that oxyresveratrol reduced cyclin A and cdk2 levels, which had an effect on S-cell cycle arrest. Oxyresveratrol-induced inhibition of cyclin A and cdk2 likely resulted in decreased progression through the $\mathrm{S}$ phase.

Although cells can die through non-apoptotic mechanisms, an intact apoptotic pathway is the most common and preferred route, and induction of apoptosis is necessary for many chemotherapeutic agents (Aleo et al., 2006). In addition, the major mechanism of several polyphenols leading to cancer cell death is apoptosis (Miura et al., 2008; Liu et al., 2009; Yoon and Liu, 2007; Zheng et al, 2013). Oxyresveratrol increased early apoptotic cells and late apoptotic cells in NCI-H520 cells based on percentages of Annexin V-positive staining (Figure 1c) and sub$\mathrm{G}_{1}$ phase cells (Figure 1b), respectively. Accordingly, cells were treated with oxyresveratrol for $24 \mathrm{~h}$, and then cyclin D was also suppressed (Figure 2), indicating that oxyresveratrol inhibited progression of the $\mathrm{G}_{0} / \mathrm{G}_{1}$ phase and resulted in enhanced cell numbers at the sub- $\mathrm{G}_{1}$ phase. Overall, these data show that apoptosis was involved in the response of NCI-H520 cells to oxyresveratrol treatment.

Apoptosis is a programmed form of cell death and can be generally divided into an extrinsic (death receptor-mediated) or intrinsic (mitochondrial) pathway. In the intrinsic signaling pathway, oxyresveratrol treatment led to the processing of caspases-3 and -9 in dose-dependent manners $(p<0.05)$ (Figure 3$)$. In order to elucidate whether oxyresveratrol activates the mitochondrial pathway, we examined the valence between proapoptotic and antiapoptotic expressions. Oxyresveratrol decreased Bcl-2 expression, an antiapoptotic protein, but did not affect Bax protein, a proapoptotic protein (Figure $3 b$ ). In addition, loss of the MMP is an indicator of apoptosis (Liu et al, 2017). Therefore, JC-1 fluorescence dye can be used as an indicator to evaluate the permeability of mitochondria membranes in NCI-H520 cells treated with oxyresveratrol. As shown in Figure 3a, treatment with oxyresveratrol significantly increased the intensity of green fluorescence in NCI-H520 cells in a dose-dependent manner, suggesting that oxyresveratrol induced reductions in the MMP. Therefore, oxyresveratrol activated the intrinsic apoptotic pathway by altering the expression level of the $\mathrm{Bcl}-2$ protein embedded in the mitochondrial membrane, inducing mitochondrial potential loss, and activating caspases-9 and -3 in NCI-H520 cells. Our study has elucidated a novel anticancer mechanism of oxyresveratrol in NCI-H520 cancer cells of inducing S phase arrest and apoptosis in an in vitro test. In addition, our study also found that oxyresveratrol can inhibit NCI-H520 tumor growth in nude mice by an i.p. injection (Figure 5).

In conclusion, our results indicated that oxyresveratrol's suppression of cancer cell growth is mainly achieved through the mechanisms of apoptosis and suppressing cell proliferation. Furthermore, oxyresveratrol induced NCI-H520 cell apoptosis in an intrinsic signaling pathway through increasing caspase-3 and -9 activities, decreasing the Bcl-2/Bax ratio, and causing the MMP to collapse. In addition, oxyresveratrol-mediated inhibition of cell proliferation was due to inducing S-phase arrest through downregulating expression levels of cyclin A, cyclin D, and cdk2. These results indicated that oxyresveratrol inhibited proliferation and induced apoptosis by activating an intrinsic pathway in NCI-H520 non-small cell lung cancer cells. Therefore, the study provides convincible data to demonstrate a new mechanistic pathway of oxyresveratrol against human non-small-cell lung cancer, further research is merited, and oxyresveratrol and its derived compounds can be applied as alternative remedies for human non-small-cell lung cancer.

\section{Acknowledgments}

This study was financially supported by the iEGG and Animal Biotechnology Center from The Feature Areas Research Center Program within the framework of the Higher Education Sprout Project by the Ministry of Education (MOE-107-S-0023-E) in Taiwan and TTMHH-NCHULS07005 from the National Chung-Hsing University/Tungs' Taichung MetroHarbor Hospital

\section{Conflict of interest}

The authors declare that they have no competing interests.

\section{Ethics approval and consent to participate}

All experimental procedures were approved by the Institutional Animal Care and Use Committee of National Chung Hsing University (IACUC no. 106-046).

\section{References}

Alberg, A.J., Brock, M.V., and Samet, J.M. (2005). Epidemiology of lung cancer: looking to the future. J Clin Oncol. 23: 3175-3185.

Aleo, E., Henderson, C.J., Fontanini, A., Solazzo, B., and Brancolini, C. (2006). Identification of new compounds that trigger apoptosomeindependent caspase activation and apoptosis. Cancer res. 66: 92359244.

Ban, J.Y., Jeon, S.Y., Nguyen, T.T., Bae, K., Song, K.S., and Seong, Y.H. (2006). Neuroprotective effect of oxyresveratrol from smilacis chinae rhizome on amyloid beta protein (25-35)-induced neurotoxicity in cultured rat cortical neurons. Biol Pharm Bull. 29: 2419-2424.

Breuer, C., Wolf, G., Andrabi, S.A., Lorenz, P., and Horn, T.F. (2006). Bloodbrain barrier permeability to the neuroprotectant oxyresveratrol. Neurosci Lett. 393: 113-118.

Carney, D.N. (2002). Lung cancer - time to move on from chemotherapy. N Engl J Med. 346: 126-128.

Chen, Y.C., Tien, Y.J., Chen, C.H., Beltran, F.N., Amor, E.C., Wang, R.J., Wu, D.J., Mettling, C., Lin, Y.L., and Yang, W.C. (2013). Morus alba and active compound oxyresveratrol exert anti-inflammatory activity via inhibition of leukocyte migration involving MEK/ERK signaling. BMC Complement Altern Med. 13: 45

Ettinger, D.S., Akerley, W., Borghaei, H., Chang, A.C., Cheney, R.T., Chirieac, L.R., D’Amico, T.A., Demmy, T.L., Govindan, R., Grannis, F.W. Jr, Grant, S.C., Horn, L., Jahan, T.M., Komaki, R., Kong, F.M., Kris, M.G., Krug, L.M., Lackner, R.P., Lennes, I.T., Loo, B.W. Jr, Martins, R., Otterson, G.A., Patel, J.D., Pinder-Schenck, M.C., Pisters, K.M., Reckamp, K., Riely, G.J., Rohren, E., Shapiro, T.A., Swanson, S.J., Tauer, K., Wood, D.E., Yang, S.C., Gregory, K., and Hughes, M. (2013). Non-small cell lung cancer, version 2.2013. J Natl Compr Canc Netw. 11: 645-653.

Galindo, I., Hernáez, B., Berná, J., Fenoll, J., Cenis, J.L., Escribano, J.M., and Alonso, C. (2011). Comparative inhibitory activity of the stilbenes resveratrol and oxyresveratrol on African swine fever virus replication. Antiviral Res. 91: 57-63.

George Rosenker, K.M., Paquette, W.D., Johnston, P.A., Sharlow, E.R., Vogt, A., Bakan, A., Lazo, J.S., and Wipf, P. (2015). Synthesis and biological evaluation of 3 -aminoisoquinolin-1 $(2 \mathrm{H})$-one based inhibitors of the dual-specificity phosphatase Cdc25B. Bioorg Med Chem. 23: 2810-2818.

Heist, R.S., Mino-Kenudson, M., Sequist, L.V., Tammireddy, S., Morrissey, L., Christiani, D.C., Engelman, J.A., and lafrate, A.J. (2012). FGFR1 amplification in squamous cell carcinoma of the lung. J Thorac Oncol. 7: 1775-1780.

Hu, C.Q., Chen, Z.D., Yao, R.F., and Xu, G. (2016). Inhibition of protein kinade $\mathrm{C}$ by stilbene derivatives from Morus alba $\mathrm{L}$. Nat Prod Res Dev 
8: 13-16.

Jagtap, U.B., and Bapat, V.A. (2010). Artocarpus: A review of its traditional uses, phytochemistry and pharmacology. J Ethnopharmacol. 129: 142-166.

Karna, P., Gundala, S.R., Gupta, M.V., Shamsi, S.A., Pace, R.D., Yates, C. Narayan, S., and Aneja, R. (2011). Polyphenol-rich sweet potato greens extract inhibits proliferation and induces apoptosis in prostate cancer cells in vitro and in vivo. Carcinogenesis. 32: 1872-1880.

Kwon, S.B., Kim, M.J., Ham, S.Y., Park, G.W., Choi, K.D., Jung, S.H., and Yoon, D.Y. (2015). H9 induces apoptosis via the intrinsic pathway in nonsmall-cell lung cancer A549 cells. J Microbiol Biotechnol. 25: 343-352.

Levy, M.A., and Claxton, D.F. (2017). Therapeutic inhibition of BCL-2 and related family members. Expert Opin Investig Drugs. 26: 293-301.

Li, H., Wu, W.K., Zheng, Z., Che, C.T., Li, Z.J., Xu, D.D., Wong, C.C., Ye, C.G., Sung, J.J., Cho, C.H., and Wang, M. (2010). 3,3',4,5,5'-Pentahydroxytrans-stilbene, a resveratrol derivative, induces apoptosis in colorectal carcinoma cells via oxidative stress. Eur J Pharmacol. 637: 55-61.

Li, Y.R., Li, S., Ho, C.T., Chang, Y.H., Tan, K.T., Chung, T.W., Wang, B.Y., Chen, Y.K., and Lin, C.C. (2016). Tangeretin derivative, 5-acetyloxy-6,7,8,4' tetramethoxyflavone induces $\mathrm{G} 2 / \mathrm{M}$ arrest, apoptosis and autophagy in human non-small cell lung cancer cells in vitro and in vivo. Cancer Biol Ther. 17: 48-64.

Liu, J.R., Dong, H.W., Chen, B.Q., Zhao, P., and Liu, R.H. (2009). Fresh apples suppress mammary carcinogenesis and proliferative activity and induce apoptosis in mammary tumors of the Sprague-Dawley rat. J Agric Food Chem. 57: 297-304.

Liu, Z., Ren, B., Wang, Y., Zou, C., Qiao, Q., Diao, Z., Mi, Y., Zhu, D., and Liu, $X$. (2017). Sesamol induces human hepatocellular carcinoma cells apoptosis by impairing mitochondrial function and suppressing autophagy. Sci Rep. 7: 45728.

Lorenz, P., Roychowdhury, S., Engelmann, M., Wolf, G., and Horn, T.F. (2003). Oxyresveratrol and resveratrol are potent antioxidants and free radical scavengers: effect on nitrosative and oxidative stress derived from microglial cells. Nitric Oxide. 9: 64-76.

Middleton, E. Jr, Kandaswami, C., and Theoharides, T.C. (2000). The effects of plant flavonoids on mammalian cells: implications for inflammation, heart disease, and cancer. Pharmacol Rev. 52: 673-751.

Miura, T., Chiba, M., Kasai, K., Nozaka, H., Nakamura, T., Shoji, T., Kanda, T. Ohtake, Y., and Sato, T. (2008). Apple procyanidins induce tumor cell apoptosis through mitochondrial pathway activation of caspase-3. Carcinogenesis. 29: 585-593

Molina, J.R., Yang, P., Cassivi, S.D., Schild, S.E., and Adjei, A.A. (2008). Nonsmall cell lung cancer: epidemiology, risk factors, treatment, and survivorship. Mayo Clin Proc. 83: 584-594.

Mouihate, A., Horn, T.F., and Pittman, Q.J. (2006). Oxyresveratrol dampens neuroimmune responses in vivo: A selective effect on TNF- $\alpha$. Am J Physiol Regul Integr Comp Physiol. 291: R1215-R1221.

Nimmanpisut, S., Chudapongse, P., and Ratanabanangkoon, K. (1976). Effects of 2,4,3,5-tetrahydroxystilbene on oxidative phosphorylation by rat liver mitochondria. Biochem Pharmacol. 25: 1245-1248.

Nishizuka, Y. (1984). The role of protein kinase $C$ in cell surface signal transduction and tumour promotion. Nature 308: 693-698.

Oh, H., Ko, E.K., Jun, J.Y., Oh, M.H., Park, S.U., Kang, K.H., Lee, H.S., and Kim, Y.C. (2002). Hepatoprotective and free radical scavenging activities of prenylflavonoids, coumarin, and stilbene from Morus alba. Planta Med. 68: 932-934.
Priyadarsini, R.V., and Nagini, S. (2012). Cancer chemoprevention by dietary phytochemicals: promises and pitfalls. Curr Pharm Biotechnol. 13: $125-136$.

Qiu, F., Komatsu, K., Saito, K., Kawasaki, K., Yao, X., and Kano, Y. (1996). Pharmacological properties of traditional medicines. XXII. Pharmacokinetic study of mulberroside $A$ and its metabolites in rat. Biol Pharm Bull. 19: 1463-1467.

Sant, M., Aareleid, T., Berrino, F., Bielska Lasota, M., Carli, P.M., Faivre, J., Grosclaude, P., Hédelin, G., Matsuda, T., Møller, H., Möller, T., Verdecchia, A., Capocaccia, R., Gatta, G., Micheli, A., Santaquilani, M., Roazzi, P., and Lisi, D. (2003). EUROCARE-3: survival of cancer patients diagnosed 1990-94-results and commentary. Ann Oncol. 5: S61-118.

Shi, Y. (2002). Mechanisms of caspase activation and inhibition during apoptosis. Mol Cell. 9: 459-470.

Shin, N.H., Ryu, S.Y., Lee, H., Min, K.R., and Kim, Y. (1998). Inhibitory effects of hydroxystilbenes on cyclooxygenase from sheep seminal vesicles. Planta Med. 64: 283-284.

Shukla, S., Meeran, S.M., and Katiyar, S.K. (2014). Epigenetic regulation by selected dietary phytochemicals in cancer chemoprevention. Cancer letters. 355: 9-17.

Song, S., Lee, H., Jin, Y., Ha, Y.M., Bae, S., Chung, H.Y., and Suh, H. (2007). Syntheses of hydroxy substituted 2-phenyl-naphthalenes as inhibitors of tyrosinase. Bioorganic Med Chem Lett. 17: 461-464.

Su, B.N., Cuendet, M., Hawthorne, M.E., Kardono, L.B., Riswan, S., Fong, H.H., Mehta, R.G., Pezzuto, J.M., and Kinghorn, A.D. (2002). Constituents of the bark and twigs of Artocarpus dadah with cyclooxygenase inhibitory activity. J Nat Prod. 65: 163-169.

Surh, Y.J (2003). Cancer chemoprevention with dietary phytochemicals. Nat Rev Cancer 3: 768-780.

Tan, H., Gao, S., Zhuang, Y., Dong, Y., Guan, W., Zhang, K., Xu, J., and Cui, J.R.-. (2016). Phycoerythrin induces SGC-7901 apoptosis by arresting cell cycle at $S$ phase. Mar Drugs. 14: E166.

Tilaoui, M., Mouse, H.A., Jaafari, A., and Zyad, A. (2014). Differential effect of artemisinin against cancer cell lines. Nat Prod Bioprospect. 4: 189-196.

Toh, C.K., Gao, F., Lim, W.T., Leong, S.S., Fong, K.W., Yap, S.P., Hsu, A.A., Eng, P., Koong, H.N., Thirugnanam, A., and Tan, E.H. (2006). Never-smokers with lung cancer: epidemiologic evidence of a distinct disease entity. J Clin Oncol. 24: 2245-2251.

Watson, W.H., Cai, J., and Jones, D.P. (2000). Diet and apoptosis. Annu Rev Nutr. 20: 485-505.

Wu, L.S., Wang, X.J., Wang, H., Yang, H.W., Jia, A.Q., and Ding, Q. (2010). Cytotoxic polyphenols against breast tumor cell in Smilax china L. J. Ethnopharmacol. 130: 460-464.

Xu, L., Liu, C., Xiang, W., Chen, H., Qin, X., and X, H.u.a.n.g. (2014). Advances in the study of oxyresveratrol. Int J Pharm. 10: 44-54.

Yang, C.S., Landau, J.M., Huang, M.T., and Newmark, H.L. (2001). Inhibition of carcinogenesis by dietary polyphenolic compounds. Annu. Rev. Nutr. 21: 381-406.

Yoon, H., and Liu, R.H. (2007). Effect of selected phytochemicals and apple extracts on NF-kappaB activation in human breast cancer MCF-7 cells. J Agric Food Chem. 55: 3167-3173.

Zheng, C.Q., Qiao, B., Wang, M., and Tao, Q. (2013). Mechanisms of apple polyphenols-induced proliferation inhibiting and apoptosis in a metastatic oral adenoid cystic carcinoma cell line. Kaohsiung J Med Sci. 29: 239-245 